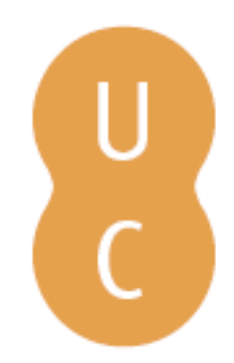

\title{
nombalina
}

\section{La reconnaissance de l'expression faciale des émotions}

\author{
Autor(es): $\quad$ Gosselin, Pierre
}

Publicado por: Imprensa da Universidade de Coimbra; Les Éditions de L'IQRC (Les

URL

persistente: URI:http://hdl.handle.net/10316.2/32644

DOI: $\quad$ DOI:http://dx.doi.org/10.14195/978-989-26-0805-1_5

Accessed : $\quad$ 26-Apr-2023 11:48:28

A navegação consulta e descarregamento dos títulos inseridos nas Bibliotecas Digitais UC Digitalis, UC Pombalina e UC Impactum, pressupõem a aceitação plena e sem reservas dos Termos e Condições de Uso destas Bibliotecas Digitais, disponíveis em https://digitalis.uc.pt/pt-pt/termos.

Conforme exposto nos referidos Termos e Condições de Uso, o descarregamento de títulos de acesso restrito requer uma licença válida de autorização devendo o utilizador aceder ao(s) documento(s) a partir de um endereço de IP da instituição detentora da supramencionada licença.

Ao utilizador é apenas permitido o descarregamento para uso pessoal, pelo que o emprego do(s) título(s) descarregado(s) para outro fim, designadamente comercial, carece de autorização do respetivo autor ou editor da obra.

Na medida em que todas as obras da UC Digitalis se encontram protegidas pelo Código do Direito de Autor e Direitos Conexos e demais legislação aplicável, toda a cópia, parcial ou total, deste documento, nos casos em que é legalmente admitida, deverá conter ou fazer-se acompanhar por este aviso.

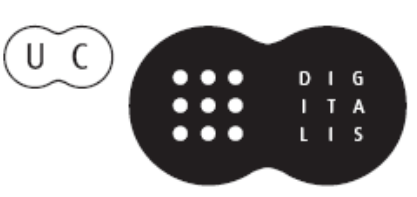


GILLES KIROUAC

Coordonnateur Scientifique

Cognition
et Émotions 
(Página deixada propositadamente em branco) 


\title{
GILLES KIROUAC
}

Coordonnateur Scientifique

\section{Cognition et Émotions}

\author{
AUTEURS \\ Klaus R. Scherer \& Janique Sangsue \\ Pierre Philippot \\ Dario Galati \\ Bernard Rimé \\ Pierre Gosselin \\ Ursula Hess \\ Arvid Kappas \& Jean Descôteaux \\ Pio E. Ricci Bitti \\ Pedro Luzes \\ Lise Fillion \\ Ramon Bayés \\ Coimbra - Imprensa da Universidade


COORDENAÇÃO EDITORIAL

Imprensa da Universidade de Coimbra

CONCEPÇÃO GRÁFICA

António Barros

PAGINAÇÃo

António Resende

[Universidade de Coimbra]

EXECUÇÃO GRÁFICA

G.C. - Gráfica de Coimbra, Lda.

Palheira - Assafarge - Apart. 3068

300I-453 Coimbra Codex

ISBN 972-8704-19-4

ISBN 2-7637-81।8-7

DEPÓSITO LEGAL 210430/04

(C) MAIO 2004, Imprensa DA UnIVERSIDAdE DE COIMBRA

\author{
OBRA PUBLICADA COM O PATROCINIO DO \\ INSTITUTO DE PSICOLOGIA COGNITIVA \\ FCT: FUNDAÇÃO PARA A CIÊNCIA E A TECNOLOGIA \\ MINISTÉRIO DA CIÊNCIA E DO ENSINO SUPERIOR \\ apolo do Programa Operacional CiênCIa, TeCnologia, Inovação \\ DO QUADRO COMUNITÁRIO DE APOIO
}


Pierre Gosselin

École de Psychologie, Université d'Ottawa

\section{LA RECONNAISSANCE DE L'EXPRESSION FACIALE DES ÉMOTIONS}

\section{INTRODUCTION}

Quels sont les développements récents qui ont nourri la réflexion dans le domaine de la reconnaissance de l'expression faciale des émotions? Trois questions nous sont apparues recevoir au cours des dernières années une attention particulière. Les deux premières font référence, en raison de leur ampleur, à des problématiques persistantes dans le domaine de l'émotion. Il s'agit de la question de la structure de l'information fournie par le comportement expressif et de celle de la portée universelle de cette information. Ces deux questions ont fait l'objet de débats théoriques récents et il nous apparait important de les évoquer. La troisième question concerne l'appréciation de l'authenticité de l'expression émotionnelle. Les travaux des dernières années ont permis de mieux documenter les mécanismes de régulation des comportements expressifs, comme le masquage, l'atténuation, l'amplification et la simulation des émotions. Dans le cadre de notre recension des travaux, nous examinerons la qualité des jugements dans l'appréciation de l'authenticité de l'expression ainsi que les facteurs qui la déterminent.

\section{LA STRUCTURE DE L'INFORMATION FOURNIE PAR L'ACTIVITÉ DU VISAGE}

L'une des grandes questions fondamentales de la psychologie de l'émotion concerne la structure des phénomènes qui sont désignés par ce terme (Frijda, 1986). Ce problème est celui qui a probablement le plus posé obstacle à l'étude psychologique des émotions. La raison principale semble 
en être la complexité des phénomènes couverts sous le terme émotion ainsi que la difficulté de les situer par rapport à ceux qui sont englobés dans le domaine, encore plus vaste, de l'affectivité.

Les discussions théoriques qui ont entouré cette question ont été stimulées récemment par une série d'articles publiés par Ortony et Turner (1990), Ekman (1992) et Izard (1992). Dans leur article, Ortony et Turner (1990) mettent en question l'existence des émotions fondamentales postulées par les théories psychoévolutionnistes. La critique des auteurs porte sur l'appui empirique de la primauté biologique et psychologique des émotions fondamentales, sur le caractère irréductible de celles-ci, sur le nombre d'émotions fondamentales et sur la possibilité d'expliquer la diversité de la vie affective à partir des émotions fondamentales. Cet article a été suivi d'une vive réaction des théoriciens psychoévolutionnistes (Ekman, 1992; Izard, 1992) qui ont fait valoir l'ampleur des données empiriques en faveur de l'existence des émotions fondamentales.

Selon la perspective psychoévolutionniste (Ekman, 1977, 1984, 1992; Izard, 1977, 1990; Plutchik, 1980; Tomkins, 1982), la vie émotionnelle est un tissu complexe composé d'émotions fondamentales et de structures affectives-cognitives. Les émotions fondamentales sont des catégories discrètes d'émotions et chacune de celles-ci se caractérise par un patron expressif, physiologique et phénoménologique qui lui est propre. De plus, chacune d'elles possède une fonction adaptative qui se présente sous la forme d'une tendance à l'action. L'expression et la reconnaissance des émotions fondamentales jouent un rôle important dans la régulation du comportement social de l'espèce. Elles favorisent la cohésion sociale et permettent une réduction des conflits interindividuels. Sept émotions fondamentales font l'objet d'un fort consensus parmi les théoriciens psychoévolutionnistes : la joie, la peur, la colère, la surprise, la tristesse, le dégoût et le mépris.

Dans leur plaidoyer en faveur des émotions fondamentales, Ekman (1992) et lzard (1992) font ressortir divers arguments qui attestent leur fondement biologique. Premièrement, il existe des changements physiologiques, notamment au niveau des systèmes nerveux périphérique et central, qui caractérisent les émotions fondamentales. Deuxièmement, les émotions fondamentales sont caractérisées par des expressions faciales qui sont universelles. Troisièmement, ces expressions faciales émergent au cours des premiers mois de la vie. Quatrièmement, ces émotions existent aussi chez 
d'autres primates. Cinquièmement, bien que la question du nombre exact d'émotions fondamentales demeure ouverte, le désaccord entre les théoriciens psychoévolutionnistes est moindre que ce qui est affirmé par Ortony et Turner (1990). II existe un fort consensus pour au moins six à sept émotions fondamentales.

Nous évoquons ici cette controverse sur l'existence des émotions fondamentales parce qu'elle est éminemment pertinente au domaine de la reconnaissance de l'expression émotionnelle. Elle nous conduit à nous questionner sur la structure de l'information fournie par les canaux expressifs.

Ekman (1992) et lzard (1992) présentent plusieurs arguments montrant que les canaux expressifs communiquent des émotions fondamentales. Ils présentent aussi plusieurs données montrant que certaines expressions faciales émotionnelles sont reconnues universellement. Cependant, ces données ne représentent que les premiers jalons d'une réponse à la question générale de la structure de l'information fournie par les canaux expressifs. Plusieurs questions plus spécifiques demeurent ouvertes. Ainsi, les caractéristiques des réponses expressives qui permettent aux décodeurs de les interpréter comme des émotions fondamentales sont encore peu documentées. Existe-t-il plusieurs expressions qui permettent de communiquer une même émotion fondamentale? Si oui, ces expressions communiquent-elles quelque chose de plus spécifique sur l'émotion? II est enfin une autre question qui demeure ouverte: celle de la valeur du concept de prototype dans l'étude de la reconnaissance des expressions émotionnelles. Dans les paragraphes qui suivent, nous exposerons et discuterons les résultats des études récentes qui ont tenté d'apporter une réponse à ces questions.

\section{La richesse du répertoire expressif}

L'idée selon laquelle une émotion fondamentale peut être communiquée par plusieurs expressions différentes a été suggérée par Ekman et Friesen (1978, 1986). Sur la base d'anályses rétrospectives de leurs études inter culturelles, ces auteurs ont proposé un répertoire facial des émotions fondamentales. Au total, près d'une centaine expressions sont comprises dans ce répertoire. La diversité des expressions varie selon l'émotion. On ne compte que quelques expressions pour le mépris et la joie, autour d'une 
dizaine pour la surprise et le dégoût, et au delà d'une vingtaine pour la peur, tristesse et la colère.

L'évaluation systématique des propositions d'Ekman et Friesen n'a été entreprise que très récemment. Les premières indications ont été rapportées par Wiggers (1982) qui a examiné la reconnaissance d'une dizaine d'expressions du répertoire ainsi que certaines de leurs variantes. Son étude fait mention d'un niveau élevé d'exactitude pour les expressions de joie, de colère, de surprise et de dégoût. Par contre, l'exactitude des jugements fut plutôt faible pour les expressions de peur et intermédiaire pour celles de la tristesse.

Plus récemment, Gosselin et Kirouac (1995) ont examiné la reconnaissance d'une trentaine d'expressions du répertoire d'Ekman et Friesen (1978). Les résultats montrèrent que les expressions de joie, de colère, de surprise et de tristesse furent très bien reconnues. Des résultats plus partagés furent cependant obtenus dans les cas de la peur et du dégoût. Seulement deux des six expressions de dégoût examinées furent reconnues avec une forte exactitude et aucune des six expressions de peur examinées ne fut fortement reconnue.

Les données actuelles portent donc à croire que plusieurs expressions peuvent communiquer une même émotion fondamentale. L'examen du répertoire facial est cependant loin d'être complété et d'autres recherches devront être conduites pour mieux apprécier la diversité des expressions qui peuvent communiquer les émotions fondamentales.

\section{La spécificité de l'information fournie par les expressions émotionnelles}

Le fait que plusieurs expressions peuvent communiquer une même émotion fondamentale nous conduit à la deuxième question que nous avons formulée plus haut: ces expressions communiquent-elles quelque chose de plus spécifique sur l'émotion? Les données disponibles sur cette question se limitent actuellement aux expressions du dégoût.

A l'intérieur d'une série de trois études, Rozin, Lowery et Ebert (1994) ont examiné l'interprétation que des décodeurs faisaient de neuf expressions faciales de dégoût. Plus spécifiquement, les décodeurs devaient choisir l'expression du visage qui correspondait le mieux à la réaction d'une personne dans diverses situations. Plus d'une cinquantaine de situations 
étaient décrites aux participants. Elles faisaient référence à des stimulations sensorielles désagréables ou irritantes (gustatives, olfactives et visuelles), à des pratiques sexuelles déviantes, à des pratiques hygiéniques malsaines, à des contaminations inter personnelles et à des offenses morales.

Les résultats de l'étude montrèrent que les expressions impliquant le plissement de la peau du nez était principalement associé à des stimulations olfactives désagréables, que l'ouverture de la bouche et l'extension de la langue étaient associées à des stimulations gustatives irritantes et que le relèvement de la lèvre supérieure était associé principalement à des situations sociales déplaisantes ou répugnantes (pratiques hygiéniques malsaines, relations sexuelles déviantes, offenses morales). L'étude de Rozin et al. (1994) appuie donc l'idée que les différentes expressions faciales d'une émotion fondamentale peuvent communiquer une information plus spécifique sur la nature de la situation qui provoque cette émotion.

\section{La notion de prototype facial}

La notion de prototype facial a été proposée pour désigner les expressions faciales qui sont les plus représentatives de chacune des émotions fondamentales et qui sont reconnues inter culturellement (Ekman \& Friesen, 1978; Ekman, Levenson \& Friesen, 1983). Bien que cette notion ait été proposée depuis plus d'une vingtaine d'années, les travaux de recherche n'ont pas encore permis de documenter de façon satisfaisante l'étendue et l'organisation de chacune des classes expressives. Les travaux de Wiggers (1982) et de Gosselin et Kirouac (1995) indiquent que plusieurs des prototypes proposés par Ekman et Friesen (1978) sont fortement reconnus par des observateurs. Cependant, cet appui empirique à la notion de prototype demeure assez modeste puisque rien ne garantit que d'autres configurations faciales que celles étudiées dans ces études ne seraient pas mieux reconnues. II conviendrait dans les années futures de mieux délimiter l'étendue des combinaisons d'unités d'action qui sont interprétées comme étant représentatives de chacune des émotions fondamentales et d'identifier parmi elles celles qui génèrent les niveaux de reconnaissance les plus élevés.

Par ailleurs, une meilleure connaissance des prototypes faciaux exigera que la structure même de ces signaux, et non seulement l'interprétation à 
laquelle ils peuvent donner lieu, soit examinée plus en détail. L'universalité de la reconnaissance de certaines configurations faciales a constituée jusqu'à présent la principale évidence de l'existence de prototypes. Le critère de reconnaissance ne pourrait cependant suffire à lui seul pour établir que les configurations en question sont effectivement émotionnelles. II se pourrait que les configurations faciales que des observateurs indépendants identifient comme étant les plus représentatives des émotions soient en fait des entités idéales, des constructions de l'appareil cognitif, et qu'elles ne sont jamais ou rarement observées dans la réalité. Cette interprétation a été proposée récemment par Carroll et Russell (1997) pour expliquer le fait que les prototypes faciaux sont rarement observés lorsque des émotions sont exprimées. Selon ces auteurs, les patrons d'activité faciale qui accompagnent les émotions seraient très variables, impliqueraient majoritairement des combinaisons d'un nombre réduit d'unités d'action et se caractériseraient par une structure componentielle (Scherer, 1984; Smith \& Scott, 1997). Référant aux divers travaux réalisés en sciences cognitives sur la formation des prototypes (Nosofsky, 1992; Posner \& Keele, 1968), Carroll et Russell émettent l'hypothèse que les prototypes faciaux correspondraient à des abstractions dans lesquelles se trouvent combinées un éventail plus large d'unités d'action que ce qui est observé dans la vie quotidienne. Une telle critique fait ressortir les limites des connaissances qui peuvent être acquises à partir des études de jugement et la nécessité d'étudier plus intensivement la structure physique des signaux émotionnels émis par l'être humain.

\section{L'UNIVERSALITÉ DE LA RECONNAISSANCE DE L'EXPRESSION FACIALE DES ÉMOTIONS}

L'universalité de l'information fournie par les canaux expressifs est la deuxième question qui a suscité de vives discussions au cours des dernières années. L'importance qu'on lui accorde est liée au fait qu'elle se rattache à la question plus large des déterminants biologiques de l'émotion.

Selon les théories psychoévolutionnistes (Ekman, 1977, 1984; Izard, 1977. 1990; Plutchik, 1980), l'expression spontanée des émotions résulte de l'activation d'un programme déterminé génétiquement. L'une des implications de ce déterminisme est qu'il existe un répertoire expressif commun à l'espèce humaine. Dans la mesure où ce répertoire existe, l'information qu'il 
véhicule doit avoir une portée transculturelle. Ceci dit, les versions récentes des théories psychoévolutionnistes admettent l'existence d'un déterminisme culturel, lequel affecterait la régulation du comportement émotionnel ainsi que la conceptualisation des émotions.

A ce déterminisme biologique, s'est opposée historiquement la thèse relativiste, principalement soutenue par des anthropologues, des sociologues et des psychologues sociaux (Birdwhistell, 1970; Harré, 1986; Lutz, 1982). La thèse relativiste met l'accent sur les variations culturelles qui caractérisent le comportement émotionnel. Les versions les plus extrêmes de cette thèse affirment que le comportement émotionnel est essentiellement un produit culturel, modelé par les valeurs, les croyances et l'organisation particulière d'un groupe social. Deux prédictions sont autorisées à partir de cette perspective. Premièrement, la reconnaissance des expressions émotionnelles doit se caractériser par l'existence de niveaux de compétences inégaux entre les cultures. En particulier, les individus d'un groupe social donné devraient mieux reconnaitre leurs propres expressions que celles des autres groupes. Deuxièmement, les niveaux de reconnaissance des expressions des autres groupes sociaux ne devraient être supérieurs au hasard que dans la mesure où l'apprentissage des normes culturelles de ces autres groupes a été possible. Les groupes sociaux isolés ne devraient pas pouvoir reconnaître les expressions émotionnelles des autres groupes.

La question de l'universalité de l'information fournie par les canaux expressifs a fait l'objet de vastes programmes de recherche inter culturelle à partir de la fin des années soixante (Ekman, Sorenson \& Friesen, 1969; Ekman et Friesen, 197I; Izard, 197I) et plusieurs recherches d'envergure ont eu lieu récemment (Ekman \& Friesen, 1986; Ekman et al., 1987; Haidt \& Kelner, 1999; Matsumoto \& Ekman, 1989, Russell, Lewicka \& Niit, 1989). En raison des difficultés méthodologiques rencontrées dans la description même du comportement expressif, la question de l'universalité des expressions émotionnelles a été principalement examinée à partir des études de reconnaissance.

Plusieurs analyses critiques ont été consacrées à cette littérature (Ekman, 1972, Ekman, 1982; Fridlund, 1994; Izard, 1971; Mesquita \& Frijda, 1992) et certaines d'entre elles ont pris un caractère polémique (Russell, 1994; Ekman, 1994; Izard, 1994). L'intérêt que présentent ces polémiques est multiple. Elles ont conduit d'une part à une reformulation plus précise de la thèse universaliste, en corrigeant notamment certaines erreurs d'interprétation 
dont elle a fait l'objet. Elles ont donné lieu d'autre part à une évaluation critique des données empiriques qui sont disponibles sur cette question, permettant, en autres, une mise en perspective des faits d'observation les plus saillants. Enfin, elles ont permis, dans une certaine mesure, de dégager de nouvelles orientations de recherche.

\section{La thèse universaliste}

À l'intérieur de leurs écrits, les défendeurs de la position universaliste (Ekman, 1994; Izard, 1994) sont d'abord conduits à identifier et à préciser les principales propositions théoriques de la perspective psychoévolutionniste. Selon cette perspective, l'expression spontanée des émotions est le résultat de l'activation d'un programme affectif faisant suite à la perception et à l'interprétation de certaines catégories d'événements. Ce programme affectif est déterminé génétiquement et permet une réaction rapide de l'organisme.

La première conséquence de l'existence d'un répertoire émotionnel commun à l'espèce humaine est le fait qu'il doit exister une certaine quantité d'expressions émotionnelles qui sont reconnues universellement. II ne s'agit pas de toutes les expressions émotionnelles, mais seulement de celles qui correspondent aux expressions spontanées faisant partie du répertoire. La mise à l'épreuve de cette proposition nécessite donc de la part des chercheurs des efforts particuliers dans la sélection des stimuli.

La deuxième précision concerne le critère de performance qui doit être utilisé pour évaluer la reconnaissance. Formellement, la reconnaissance implique un niveau de performance supérieur au hasard, ce qui, selon Ekman (1994), serait une condition suffisante. Plusieurs facteurs font qu'on ne peut

104 s'attendre d'emblée à une reconnaissance parfaite. D'une part, la conceptualisation des émotions diffère selon les cultures, ce qui fait qu'il n'est pas aisé de trouver des termes émotionnels équivalents d'une culture à l'autre. De plus, même à l'intérieur des collectivités qui partagent la même langue, un même mot peut avoir des connotations différentes. Enfin, les difficultés techniques qui se présentent dans la réalisation des études peuvent conduire à une sous-estimation des capacités des sujets. Ce problème se pose de façon plus particulière avec les populations isolées, étrangères aux études scientifiques. 
Enfin, les défenseurs de la thèse universaliste rappellent que la perspective psychoévolutionniste ne nie pas l'influence de la culture sur l'expression et la reconnaissance des émotions. La théorie neuroculturelle (Ekman, 1977, 1984) et la théorie des émotions différentielles (Izard, 1977, 1991) contiennent des propositions spécifiques touchant l'influence du milieu sur l'induction des émotions et sur la régulation des comportements expressifs. II en découle que l'existence de niveaux différents de reconnaissance entre les cultures n'est pas un argument qui contredit la thèse universaliste. II peut exister à la fois des expressions universelles et d'autres qui relèvent des acquisitions culturelles.

\section{Convergence des observations}

Suite aux analyses critiques de la littérature que l'on retrouve dans les derniers débats théoriques (Ekman, 1994; Izard, 1994, Russell, 1994), il est permis d'identifier plusieurs faits d'observation particulièrement robustes autour desquels se dégage un consensus entre les auteurs.

\section{Les membres de plusieurs pays occidentaux ou occidentalisés parviennent à bien reconnaître les expressions faciales des membres des autres pays.}

Le premier fait d'importance, et certainement le plus documenté, concerne la reconnaissance de l'expression faciale dans les pays occidentaux ou occidentalisés. Les études menées dans plus d'une trentaine de pays, en Europe, en Amérique et en Asie, indiquent que les membres d'un pays donné réussissent à reconnaître les expressions faciales des membres des autres pays (Ekman, 1972; Ekman \& Friesen, 1986; Ekman et al. 1987; Izard, 1971). Le niveau de performance des sujets n'est pas simplement supérieur au hasard, il est élevé pour la plupart des émotions fondamentales. Ce résultat est en accord avec la thèse universaliste, mais il n'en constitue qu'un appui partiel. En effet, comme la majorité des études ont porté sur les populations de pays industrialisés ayant des échanges culturels et commerciaux entre eux, les forts pourcentages de reconnaissance pourraient aussi traduire l'apprentissage des normes culturelles. 


\section{Les membres de populations isolées sur le plan culturel parviennent à reconnaître les expressions faciales des membres des autres collectivités.}

Les données les plus décisives proviennent des études faites auprès de populations isolées sur le plan culturel, c'est à dire de groupes culturels n'ayant pas eu l'occasion de faire l'apprentissage des normes culturelles des pays occidentalisés. De tels groupes existaient toujours dans le début des années 1970, notamment en Nouvelle-Guinée et à Bornéo. Les résultats de ces études montrent que plusieurs groupes culturels isolés (Fore, Dani, Sadong, Bahinemo) parviennent à reconnaître les expressions faciales des membres de cultures occidentalisées (Ekman, Sorenson \& Friesen, 1969; Ekman \& Friesen, 1971; Sorenson, 1975, 1976). Un tel résultat appuie la position universaliste et est difficilement conciliable avec la thèse relativiste.

\section{Le niveau de reconnaissance des expressions faciales varie en fonction des groupes culturels.}

Bien que les membres d'un groupe culturel parviennent à reconnaître les expressions faciales des autres groupes culturels, plusieurs études révèlent que le niveau de performance n'est pas uniforme d'une culture à l'autre pour certaines émotions (Izard, 1971; Ekman et al. 1987, Matsumoto, 1992; Matsumoto \& Ekman, 1989). C'est le cas, plus particulièrement, de la peur, de la colère et du dégoût. Ces différences pourraient refléter l'existence de variations culturelles dans l'encodage des expressions (Markham \& Wang, 1996). Elles pourraient aussi résulter de certains biais ethniques de jugement (Kilbride \& Warczower, 1983) ou de certaines règles culturelles de décodage (Biehl et al., 1997). II est également possible que ces différences soient attribuables à l'imperfection des mesures de la reconnaissance. Les variations culturelles qui existent dans la conceptualisation et le lexique des émotions (Matsumoto \& Assar, 1992) font qu'il n'est pas aisé d'assurer l'équivalence des tâches de jugement. Quant au niveau plus faible de reconnaissance atteint par les membres des groupes culturels isolés, il pourrait traduire, selon Ekman (1994), le 
manque de familiarité de ces groupes avec le rôle de participants dans des études scientifiques.

\section{Les études de jugement impliquant un choix forcé ont généré des niveaux de reconnaissance interculturelle plus élevés que celles faisant appel à une désignation verbale libre.}

Les études ayant rapporté les niveaux de reconnaissance interculturelle les plus élevés ont soumis les décodeurs à une tâche dans laquelle ils devaient choisir, parmi plusieurs termes émotionnels proposés, celui qui correspondait à l'expression faciale. Lorsque les sujets doivent choisir le terme émotionnel qui leur vient spontanément à l'esprit, le niveau d'accord est sensiblement plus faible. Cette observation est révélatrice, selon Russell (1994), des variations culturelles dans la conceptualisation des émotions et conséquemment dans le lexique propre à chaque langue. II importe toutefois de dire que l'on retrouve cette différence entre les deux types de tâche à l'intérieur d'un même groupe culturel. Elle traduirait donc plus fondamen-talement une variabilité dans la compréhension et l'utilisation du langage (Ekman, 1994; Izard, 1994). Une étude récente de Haidt et Keltner (1999) montre par ailleurs que les jugements produits avec la désignation verbale libre conduit à des résultats très convergents avec ceux obtenus avec la méthode de choix forcé, suggérant que les forts niveaux de reconnaissance obtenus avec ce dernier type de jugement ne sont pas le produit d'un artefact méthodologique.

Cette différence entre les deux tâches de jugement est intéressante parce qu'elle souligne à quel point le niveau d'analyse choisi a un impact sur la mise en évidence des universaux. Comme le soulignent Mesquita et Frijda (1992), la question de l'universalité peut être examinée à des niveaux plus ou moins abstraits. Les évidences les plus fortes de l'existence des universaux proviennent des études impliquant un niveau de traitement plus abstrait. C'est le cas lorsque la tâche de jugement implique un choix forcé. Les décodeurs doivent alors associer une expression faciale à une catégorie émotionnelle, c'est-à-dire à une entité abstraite à laquelle se trouvent subsumées des réalités plus concrètes. Lorsque la tâche de jugement implique une désignation verbale libre, les influences culturelles et interindividuelles se manifestent davantage, introduisant une variabilité qui peut masquer l'existence des universaux. 


\section{Direction future de la recherche}

Les études de jugement ont permis d'apporter plusieurs éléments de réponse à la question de l'universalité de la reconnaissance des expressions faciales des émotions. II est toutefois un aspect de la démonstration qui requiert une meilleure documentation. II s'agit du caractère véritablement émotionnel des expressions faciales qui ont généré de forts niveaux de reconnaissance sur le plan inter culturel. Ce point est probablement le plus crucial de ceux soulevés par les critiques de la position universaliste (Fridlund, 1994; Russell, 1994). II conviendrait de démontrer que ces expressions sont valides et qu'elles ont une valeur écologique. Si elles remplissent effectivement ces conditions, des études de composantes devraient révéler qu'elles ont une forte probabilité d'apparition lorsque des membres de cultures diverses expriment spontanément une émotion. Les indications de cette nature demeurent encore aujourd'hui peu nombreuses.

\section{LE JUGEMENT DE L'AUTHENTICITÉ DE L'EXPRESSION FACIALE DES ÉMOTIONS}

Les mécanismes de contrôle des comportements expressifs contribuent grandement à complexifier le processus de communication des émotions. Le fait que l'être humain puisse atténuer, amplifier, simuler et masquer son expression émotionnelle (Ekman, 1977, 1993) augmente considérablement la difficulté d'interprétation des messages émotionnels. Les conséquences du contrôle varient en fonction des stratégies particulières employées par l'encodeur. L'atténuation et l'amplification, par exemple, peuvent conduire le décodeur à sous estimer ou à surestimer l'intensité de l'émotion. Dans les cas extrêmes d'atténuation, il est même probable que l'émotion puisse ne pas être détectée. La simulation et le masquage, de leur côté, risquent d'engendrer une interprétation tout à fait erronée de la nature de l'émotion ressentie.

Les erreurs d'interprétation susceptibles de résulter des stratégies de contrôle ne sont pas nécessairement dommageables pour le décodeur. Selon Saarni et Lewis (1993), la dissimulation de l'émotion est fréquemment utilisée pour ménager les sentiments de l'entourage social et favoriserait de la sorte des échanges sociaux plus harmonieux. La capacité à détecter la tromperie devient cependant avantageuse lorsqu'elle permet au décodeur de se soustraire à des manipulations expressément destinées à lui nuire. 
Pour juger l'authenticité de l'expression émotionnelle, les décodeurs disposent d'une certaine quantité d'indices. Ainsi, les expressions faciales simulées sont plus souvent asymétriques que ne le sont les expressions authentiques (Ekman, Hager \& Friesen, 1981; Hager \& Ekman, 1985) et elles présentent une dynamique de contraction musculaire plus irrégulière (Hess \& Kleck, 1990; Weiss, Blum \& Gleberman, 1987). De leur côté, les expressions faciales produites pour masquer une émotion se distinguent des expressions authentiques par l'absence de certaines composantes faciales. Lorsqu'une personne ressentant la peur ou le dégoût simule la joie, la contraction de l'orbicularis oculi, produisant la levée des joues et tirant la peau autour des yeux vers l'intérieur des orbites oculaires, est moins fréquente que lorsque l'expression est authentique (Ekman, Friesen \& O'Sullivan, 1988).

Que nous révèlent les travaux portant sur l'appréciation de l'authenticité des expressions émotionnelles? Une première série de travaux ont porté sur la capacité des décodeurs à distinguer entre les expressions authentiques et les expressions de masquage. Par expression de masquage, nous désignons ici l'expression émotionnelle qui est simulée alors que l'encodeur ressent une émotion différente. Dans la plupart des travaux que nous rapportons, il s'agit de la simulation d'une émotion plaisante alors que l'émotion ressentie est déplaisante.

La majorité de ces travaux indiquent que les décodeurs sont capables, dans certaines conditions, de faire la distinction entre les deux classes d'expressions (DePaulo, 1988, Ekman \& Friesen, 1974; Ekman, O'Sullivan, Friesen \& Scherer, 1991; O'Sullivan, Ekman, Friesen \& Scherer, 1985; Zuckerman, Koestner \& Collela, 1985). En général, la sensibilité des décodeurs est très modeste, les taux de réussite ne dépassant guère $60 \%$ dans une tâche où le pourcentage attribuable au hasard est de $50 \%$. Le canal de communication affecte sensiblement la qualité des jugements. Le canal facial, notamment, conduit à des jugements moins exacts que ceux produits à partir des canaux gestuel (Ekman \& Friesen, 1974) et audio (Zuckerman et al., 1985).

Des niveaux de réussite à peu près comparables ont été obtenus dans la discrimination entre les expressions simulées et authentiques. Gosselin \& Kirouac (1994) rapportent des niveaux de performance variant entre 51\% et $62 \%$, selon les canaux de communication auxquels avaient accès les décodeurs. L'infériorité du canal facial par rapport au canal audio n'a pas été reproduite dans cette étude. II est probable que cette divergence des résultats reflète une différence entre le masquage et la simulation de 
l'émotion. La présence de l'émotion ressentie, dans le masquage, pourrait interférer avec la production de l'émotion simulée et cette interférence pourrait s'exercer plus fortement sur le canal vocal.

La présence d'un biais dans le jugement de l'authenticité contribue à la modeste performance que l'on retrouve. Tant dans les tâches impliquant la discriminations entre les expressions authentiques et de masquage (DePaulo, Stone \& Lassiter, 1985; Zuckerman, Koestner, Collela \& Alton, 1984) que celles impliquant la discrimination entre les expressions authentiques et simulées (Gosselin \& Kirouac, 1994; Gosselin, Kirouac \& Doré, 1995; Hess \& Kleck, 1994), les décodeurs sont enclins à croire en l'authenticité de l'expression. La signification de ce biais demeure obscure. L'une des interprétations possibles serait qu'il reflète une croyance selon laquelle les gens sont généralement honnêtes dans la vie quotidienne. Certains pensent même qu'une telle croyance représente un postulat indispensable à l'établissement et au fonctionnement des systèmes de communication.

II existe des différences interindividuelles importantes dans l'habileté à juger l'authenticité. Certaines personnes dont la profession implique des habiletés dans ce domaine réussissent nettement mieux que la population générale. C'est le cas des agents des services secrets américains dont la performance s'est avérée nettement supérieure au personnel des autres corps d'emploi (Ekman \& O'Sullivan, 1991).

Plusieurs facteurs semblent entrer en jeu dans la réussite au jugement de l'authenticité. D'abord, l'expérience avec les contextes de tromperie et l'attention prêtée au comportement non verbal semblent déterminantes. C'est ce que suggère la supériorité des agents des services secrets par rapport aux autres corps d'emploi. De plus, on note, chez cette population particulière, que les agents les plus étroitement impliqués dans les interrogatoires réussissent mieux que ceux dont le travail comporte principalement des tâches administratives (Ekman \& O'Sullivan, 1991).

La réussite à détecter la tromperie n'est pas reliée à la confiance que les décodeurs ont en leur propre jugement. Elle est plutôt reliée à la flexibilité du décodeur à fonder son jugement sur des indices comportementaux variés. Cette flexibilité est avantageuse parce que les indices révélateurs de la tromperie ne circulent pas toujours dans le même canal mais fluctuent d'un canal à l'autre en fonction du temps. Le problème rencontré par plusieurs décodeurs est qu'ils se concentrent sur des indices qui ne sont pas révélateurs de la tromperie, comme par exemple la direction 
du regard (Hess \& Kleck, 1994). Ils ont aussi tendance à confondre les signes de nervosité avec les indices de tromperie. Comme des encodeurs peuvent à la fois exprimer honnêtement leurs émotions et manifester de la nervosité, cette confusion réduit l'exactitude de leur jugement.

Certaines conditions permettent aux décodeurs d'accroître la qualité de leur jugement. O'Sullivan, Ekman et Friesen (1988) et Frank et al. (1993) rapportent que la performance des décodeurs est meilleure lorsqu'on leur présente successivement deux expressions, l'une honnête et l'autre trompeuse, et qu'ils doivent ensuite identifier celle qui est trompeuse. On peut penser que cette condition de jugement facilite le rappel des informations pertinentes en mémoire et conduit à une analyse comparative plus complète. Les résultats rapportés par O'Sullivan et al. indiquent aussi que l'ordre de présentation des expressions affecte la performance des décodeurs. Ces derniers réussissent mieux lorsqu'ils voient d'abord l'expression authentique et ensuite l'expression trompeuse. Selon les auteurs, ce résultat serait révélateur du processus de décision habituellement utilisé par les décodeurs, processus qui consiste à prendre l'expression authentique comme référence et à évaluer les expressions trompeuses en regard de cette référence.

Le dernier aspect sur lequel nous nous pencherons concerne la possibilité d'accroître l'exactitude des jugements dans l'appréciation de l'authenticité. Zuckerman, Koestner et Collela (1985) ont examiné l'apprentissage par rétroaction sur la détection de la tromperie et rapportent un accroissement de la performance dans les trois conditions de canaux comprises dans leur étude (facial, audio et facial+audio). De plus, l'apprentissage se généralise à d'autres encodeurs que ceux visés par la rétroaction. II faut souligner toutefois que les gains sont modestes, particulièrement pour le canal facial, et que la performance des sujets, tout en excédant le niveau attribuable au hasard, demeure très imparfaite.

En conclusion, les études de jugement portant sur l'authenticité de l'expression émotionnelle sont révélatrices de deux faits principaux. En premier lieu, elles indiquent que le jugement de l'authenticité de l'expression émotionnelle en est un qui est imparfait. L'apprentissage dans ce domaine est possible, mais il implique un entraînement extensif, une sensibilité et une attention particulières aux indices verbaux et non verbaux, et de la flexibilité dans l'utilisation de ces indices. En second lieu, il apparait que le contrôle de l'expression émotionnelle est une entreprise assez réussie, permettant 
à l'encodeur, dans près de $40 \%$ à $50 \%$ des cas, de cacher à ses congénères son véritable état émotionnel.

\section{RÉFÉRENCES}

Bielh, M., Matsumoto, D., Ekman, P., Hearn, V., Heider, K., Kudoh, T., \& Ton, V. (1997). Matsumoto and Ekman's japanese and caucasian expressions of emotion (JACFEE): Reliability data and cross-national differences. Journal of Nonverbal Behavior, 21, 3-21.

Birdwhistell, R. L. (1970). Kinesics and context. Philadelphia: University of Pennsylvania Press. Carroll, J. M., \& Russell, J. A. (1997). Facial expressions in Hollywood's portrayal of emotion. Journal of Personality and Social Psychology, 72, 164-176.

DePaulo, B. M. (1988). Nonverbal aspects of deception. Journal of Nonverbal Behavior, 12, I53-161.

DePaulo, B. M., Stone, J. I., \& Lassiter, G. D. (1985). Deceiving and detecting deceit. In B. R. Schlenger (Ed.), (pp. 323-370). The self and social life. New York: McGraw-Hill.

Ekman, P. (1972). Universals and cultural differences in facial expressions of emotion. In J. Cole (Ed.), Nebraska Symposium on Motivation (pp. 207-283). Lincoln: University of Nebraska Press.

Ekman, P. (1977). Biological and cultural contribution to body and facial movement. In J. Blacking (Ed.). The anthropology of the body. San Diego: Academic Press, p. 34-84.

Ekman, P. (1982). Emotion in the human face. New York: Pergamon Press.

Ekman, P. (1984). Expression and the nature of emotion. In K. Scherer \& P. Ekman (Eds.). Approaches to emotion. Hillsdale: Erlbaum, p. 319-344.

Ekman, P. (1992). Are there basic emotions?. Psychological Review, 99, 550-553.

Ekman, P. (1993). Facial expression and emotion. American Psychologist, 48, 384-392.

Ekman, P. (1994). Strong evidence for universals in facial expressions: A reply to Russell mistaken critique. Psychological Bulletin, II 5, 268-287.

Ekman, P., \& Friesen, W.V. (197I). Constants across cultures in the face and emotion. Journal of Personality and Social Psychology, 17, 124-129.

Ekman, P., \& Friesen, W. V. (1974). Detecting deception from the body or face. Journal of Personality and Social Psychology, 29, 288-298.

Ekman, P., \& Friesen, W. V. (1978). Facial Action Coding System: Part Two. Palo Alto: Consulting Psychologists Press.

Ekman, P., \& Friesen, W.V. (1986). A new pan-cultural expression of emotion. Motivation and Emotion, 10, 159-168.

112 Ekman, P., Friesen, W.V., \& O'Sullivan, M. (1988). Smiles when lying. Journal of Personality and Social Psychology, 54, 4|4-420.

Ekman, P., Friesen, W.V., O'Sullivan, M., Chan, A., Diacoyanni-Tarlatzis, I., Heider, K., Krause, R., Lecompte, W.A., Pitcairn, T., Ricci-Bitti, P. E., Scherer, K., Tomita, M., \& Tzavaras, A. (1987). Universals and cultural differences in the judgments of facial expressions of emotion. Journal of Personality and Social Psychology, 53, 7|2-7|7.

Ekman, P., Hager, J. C., \& Friesen, W. V. (1981). The symmetry of emotional and deliberate facial actions. Psychophysiology, 18, 101-106.

Ekman, P., Levenson, R. W., \& Friesen, W. V. (1983). Autonomic nervous system activity distinguishes among emotions. Science, 221, 1208-1210.

Ekman, P. \& O'Sullivan, M. (199I). Who can catch a liar? American Psychologist, 46, 913-920. 
Ekman, P., O'Sullivan, M. B., Friesen, W. V., \& Scherer, K. R. (199I). Face, voice and body in detecting deceit. Journal of Nonverbal Behavior, I5, 125-135.

Ekman, P., Sorenson, E. R., \& Friesen, W. V. (1969). Pan-cultural elements in facial displays of emotions. Science, 164, 86-88.

Fehr, B., \& Russell, J. A. (1984). Concept of emotion viewed from a prototype perspective. Journal of Experimental Psychology: General, 113, 464-486.

Frank, M. G., Ekman, P., \& Friesen, W.V. (1993). Behavioral markers and recognizability of the smile of enjoyment. Journal of Personality and Social Psychology, 64, 83-93.

Fridlund, A. J. (1994). Human facial expression: An evolutionary view. San Diego: Academic Press.

Frijda, N. H. (1986). The emotions. New York: Cambridge University Press.

Gosselin, P., \& Kirouac, G. (1994). Effet du canal de communication sur le jugement d'émotions ressenties et simulées. Revue Canadienne des Sciences du Comportement, 26, 163-181.

Gosselin, P., \& Kirouac, G. (1995). Le décodage de prototypes émotionnels faciaux. Revue Canadienne de Psychologie Expérimentale, 49, 313-329.

Gosselin, P., Kirouac, G., \& Doré, F.Y. (1995). Components and recognition of facial expressions in the communication of emotion by actors. Journal of Personality and Social Psychology, 68, 83-96.

Hager, J. C., \& Ekman, P. (1985). The asymmetry of facial actions is consistent with models of hemispheric specialization. Psychophysiology, 22, 307-318.

Haidt, J., \& Keltner, D. (1999). Culture and facial expression: Open-ended methods find more expressions and a gradient of recognition. Cognition and Emotion, 13, 225-266.

Harré, R. M. (1986). The social construction of emotion. Oxford: Basil Blackwell.

Hess, U., \& Kleck, R. E. (1990). Differentiating emotion elicited and deliberate emotional facial expressions. European Journal of Social Psychology, 20, 369-385.

Hess, U., \& Kleck, R. E. (1994). The cues decoders use in attempting to differentiate emotion-elicited and posed facial expressions. European Journal of Social Psychology, 24, 367-38I.

Izard, C. E. (197I). The face of emotion. New York: Appleton-Century-Crofts.

Izard, C. E. (1977). Human emotions. New York: Plenum Press.

Izard, C. E. (1990). Facial expressions and the regulation of emotions. Journal of Personality and Social Psychology, 58, 487-498.

Izard, C. E. (199|). The psychology of emotion. New York: Plenum Press.

Izard, C. E. (1992). Basic emotions, relations among emotions, and emotion-cognition relations. Psychological Review, 99, 561-565.

Izard, C. E. (1994). Innate and universal facial expressions: Evidence from developmental and cross-cultural research. Psychological Bulletin, 1 15, 288-299.

Kilbride, J. E., \& Yarczower, M. (1983). Ethnic bias in the recognition of facial expressions Journal of Nonverbal Behavior, 8, 27-4I.

Lutz, C. (1982). The domain of emotion words on Ifaluk. American Ethnologist, 9, I I3- I 28.

Markham, R., \& Wang, L. (1996). Recognition of emotion by Chinese and Australian children. Journal of Cross-Cultural Psychology, 27, 616-643.

Matsumoto, D. (1992). More evidence for the universality of a contempt expression. Motivation and Emotion, 16, 363-368.

Matsumoto, D., \& Assar, M. (1992). The effects of language on judgments of universal facial expressions of emotion. Journal of Nonverbal Behavior, 16, 85-99.

Matsumoto, D., \& Ekman, P. (1989). American-Japanese cultural differences in intensity ratings of facial expressions of emotion. Motivation and Emotion, 13, 143-157. 
Mesquita, B., \& Frijda, N. H. (1992). Cultural variations in emotions: A review. Psychological Bulletin, 1 12, 179-204.

Nosofski, R. (1992). Similarity scaling and cognitive process models. Annual Review of Psychology, 43, 25-53.

Ortony, A., \& Turner, T. J. ( 1990). What's basic about basic emotions? Psychological Review, 97, $3|5-33|$.

O'Sullivan, M., Ekman, P., \& Friesen, W.V. ( 1988$)$. The effect of comparisons on detecting deceit. Journal of Nonverbal Behavior, 12, 203-214.

O'Sullivan, M., Ekman, P., Friesen, W. V., \& Scherer, K. R. (1985). What you say and how you say it:The contribution of speech content and voice quality to judgments of others. Journal of Personality and Social Psychology, 48, 54-62.

Plutchik, R. (1980). Emotion: A psychoevolutionary synthesis. New York: Harper and Row.

Posner, M. I., \& Keele, S.W. (1968). On the genesis of abstract ideas. Journal of Experimental Psychology, 77, 353-363.

Rozin, P., Lowery, L., \& Ebert, R. (1994). Varieties of disgust faces and the structure of disgust. Journal of Personality and Social Psychology, 66, 870-88I.

Russell, J. (1991). In defense of a prototype approach to emotion concepts. Journal of Personality and Social Psychology, 60, 37-47.

Russell, J. A. (1994). Is there universal recognition of emotion from facial expression? A review of the cross-cultural studies. Psychological Bulletin, 115, 102-141.

Russell, J. A., Lewicka, M., \& Niit, T. (1989). A cross-cultural study of a circumflex model of affect. Journal of Personality and Social Psychology, 57, 848-856.

Saarni, C., \& Lewis, M. (1993). Deceit and illusion in human affairs. In M. Lewis et C. Saarni (Eds.), Lying and deception in everyday life (pp. 1-29). New York: Guilford Press.

Scherer, K. R. (1984). On the nature and function of emotion: A component process approach. In K. R. Scherer \& P. Ekman (Eds.), Approaches to emotion (pp.293-3 I8). Hillsdale: Lawrence Erlbaum Associates.

Smith, C. A., \& Scott, H. S. (1997). A componential approach to the meaning of facial expressions. In J. A. Russell \& J. M. Fernandez-Dols (Eds.), The psychology of facial expression (pp. 229-254). Cambridge (UK): Cambridge University Press.

Sorenson, E. R. (1975). Culture and the expression of emotion. In T. R. Williams (Ed.), Psychological anthropology (pp. 361-372). Chicago: Aldine.

Sorenson, E. R. (1976). The edge of the forest: Land, chilhood and change in a New Guina protoagricultural society. Washington, DC: Smithonian Institution Press.

Tomkins, S. S. (1982). Affect theory. In P. Ekman (Ed.), Emotion in the human face (pp.). New York: Cambridge University Press.

Weiss, F., Blum, G. S., \& Gleberman, L. (1987). Anatomically based measurement of facial expressions in simulated versus hypnotically induced affect. Motivation and Emotion, II, 67-81.

Wiggers, M. (1982). Judgments of facial expressions of emotion predicted from facial behavior. Journal of Nonverbal Behavior, 7, $101-116$.

Zuckerman, M., Koestner, R., \& Colella, M. J. (1985). Learning to detect deception from three communication channels. Journal of Nonverbal Behavior, 9, 188-194.

Zuckerman, M., Koestner, R., Colella, M. J., \& Alton, A. O. (1984). Anchoring in the detection of deception and leakage. Journal of Personality and Social Psychology, 47, 301-31 I. 
(Página deixada propositadamente em branco) 
Série

Investigaçāo

Coimbra

Imprensa da Universidade

Les Presses de L'Université Laval

Les Editions de L'IORC

2004 